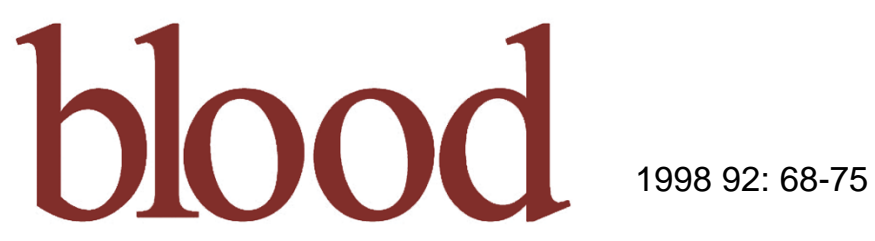

\title{
Treatment of Anemia in Myelodysplastic Syndromes With Granulocyte Colony-Stimulating Factor Plus Erythropoietin: Results From a Randomized Phase II Study and Long-Term Follow-Up of 71 Patients
}

Eva Hellström-Lindberg, Tomas Ahlgren, Yves Beguin, Magnus Carlsson, Jan Carneskog, Inger Marie Dahl, Ingunn Dybedal, Gunnar Grimfors, Lena Kanter-Lewensohn, Olle Linder, Michaela Luthman, Eva Löfvenberg, Herman Nilsson-Ehle, Jan Samuelsson, Jon-Magnus Tangen, Ingemar Winqvist, Gunnar Öberg, Anders Österborg and Ảke Öst

Updated information and services can be found at: http://bloodjournal.hematologylibrary.org/cgi/content/full/92/1/68

Articles on similar topics may be found in the following Blood collections: Clinical Trials and Observations (2501 articles)

Information about reproducing this article in parts or in its entirety may be found online at: http://bloodjournal.hematologylibrary.org/misc/rights.dt|\#repub_requests

Information about ordering reprints may be found online at:

http://bloodjournal.hematologylibrary.org/misc/rights.dt|\#reprints

Information about subscriptions and ASH membership may be found online at: http://bloodjournal.hematologylibrary.org/subscriptions/index.dtl

Blood (print ISSN 0006-4971, online ISSN 1528-0020), is published semimonthly by the American Society of Hematology, 1900 M St, NW, Suite 200, Washington DC 20036.

Copyright 2007 by The American Society of Hematology; all rights reserved.

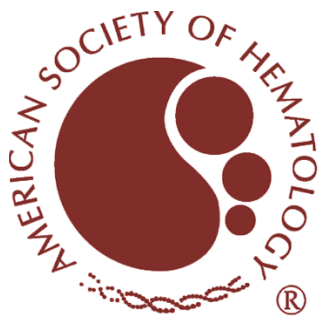




\title{
Treatment of Anemia in Myelodysplastic Syndromes With Granulocyte Colony-Stimulating Factor Plus Erythropoietin: Results From a Randomized Phase II Study and Long-Term Follow-Up of 71 Patients
}

\author{
By Eva Hellström-Lindberg, Tomas Ahlgren, Yves Beguin, Magnus Carlsson, J an Carneskog, Inger Marie Dahl, \\ Ingunn Dybedal, Gunnar Grimfors, Lena Kanter-Lewensohn, Olle Linder, Michaela Luthman, \\ Eva Löfvenberg, Herman Nilsson-Ehle, J an Samuelsson, J on-Magnus Tangen, \\ Ingemar Winqvist, Gunnar Öberg, Anders Österborg, and Åke Öst
}

\begin{abstract}
Treatment with enythropoietin (epo) may improve the anemia of myelodysplastic syndromes (MDS) in approximately $20 \%$ of patients. Previous studies have suggested that treatment with the combination of granulocyte colony-stimulating factor (G-CSF) and epo may increase this response rate. In the present phase II study, patients with MDS and anemia were randomized to treatment with G-CSF + epo according to one of two altematives; arm A starting with G-CSF for 4 weeks followed by the combination for 12 weeks, and arm B starting with epo for 8 weeks followed by the combination for 10 weeks. Fifty evaluable patients ( 10 refractory anemia [RA], 13 refractory anemia with ring sideroblasts [RARS], and 27 refractory anemia with excess blasts [RAEB]) were included in the study, three were evaluable only for epo as monotherapy and $\mathbf{4 7}$ for the combined treatment. The overall response rate to G-CSF + epo was $38 \%$, which is identical to that in our previous study. The response rates for patients with RA, RARS, and RAEB were $20 \%, 46 \%$, and $37 \%$, respec-
\end{abstract}

A PPROXIMATELY 90\% of patients with myelodysplastic syndromes (MDS) present with anemia at diagnosis and the majority of the patients develop with time a requirement for transfusions of packed red blood cells (RBC). ${ }^{1}$ In low-risk MDS, the anemia is often the only or major clinical problem and may give rise to significant morbidity. ${ }^{2}$ Quality of life is reduced due to the low hemoglobin level and in older patients, conditions such as congestive heart failure and angina pectoris are often aggravated. Moreover, repeated transfusions may with time cause secondary hemochromatosis.

The cytopenia in MDS may in some cases be ameliorated or improved by treatment with hematopoietic growth factors. Granulocyte-macrophage colony-stimulating factor (GM-CSF) and granulocyte CSF (G-CSF) are relatively effective in increasing the number of neutrophils, ${ }^{3-6}$ but have in randomized studies failed to show a positive effect on survival. Moreover, both drugs have demonstrated an overall negative effect on the

From the Department of Hematology, Huddinge University Hospital, Huddinge, Sweden; Department of Pathology, Karolinska University Hospital, Stockholm, Sweden; Department of Hematology, University of Liège, Liège, Belgium; The Scandinavian MDS Group, Sweden and Norway.

Submitted December 8, 1997; accepted February 27, 1998.

Supported by grants from the National Cancer Foundation, Stockholm, Sweden.

Address reprint requests to Eva Hellström-Lindberg, $M D, P h D$, Department of Hematology, Huddinge University Hospital, 14186 Huddinge, Sweden; e-mail: Eva.Hellstrom-Lindberg@medhs.ki.se.

The publication costs of this article were defrayed in part by page charge payment. This article must therefore be hereby marked "advertisement" in accordance with 18 U.S.C. section 1734 solely to indicate this fact.

(C) 1998 by The American Society of Hematology.

0006-4971/98/9201-0014\$3.00/0 tively. Response rates were identical in the two treatment groups indicating that an initial treatment with G-CSF was not neccessary for a response to the combination. Nine patients in arm B showed a response to the combined treatment, but only three of these responded to epo alone. This suggests a synergistic effect in vivo by G-CSF + epo. A long-term follow-up was made on 71 evaluable patients from both the present and the preceding Scandinavian study on G-CSF + epo. Median survival was 26 months, and the overall risk of leukemic transformation during a median follow-up of $\mathbf{4 3}$ months was $\mathbf{2 8 \%}$. Twenty patients entered long-term maintenance treatment and showed a median duration of response of 24 months. The intemational prognostic scoring system (IPSS) was effective to predict survival, leukemic transformation, and to a lesser extent, duration of response, but had no impact on primary response rates.

(c) 1998 by The American Society of Hematology.

platelet counts. ${ }^{7,8}$ The risk for leukemic transformation did not seem to be changed in actively treated patients. Erythropoietin (epo) is a potent stimulator of normal erythropoiesis and may also improve the anemia in patients with MDS. ${ }^{9-11}$ The efficacy of epo alone is relatively low, around $20 \%$, and mainly confined to patients without the need for pretreatment transfusion. ${ }^{12}$ In a recent meta-analysis the response rate in patients with ring sideroblastic anemia (RARS), who generally show a relatively good median survival, was only $8 \%$, while it was $21 \%$ in patients with refractory anemia (RA) and refractory anemia with excess blasts (RAEB). ${ }^{12}$ Other predictors of response were absence of the need for an initial transfusion and a serum epo level of $<200 \mathrm{U} / \mathrm{L}$.

Epo in combination with several other early-acting or myeloid cytokines has shown synergistic effects on erythropoiesis in vitro. ${ }^{13,14}$ The combination of G-CSF and epo has been administered in five clinical studies aiming at improving the anemia in MDS. These studies have mainly comprised patients with RA, RARS, and RAEB. Two of the studies ${ }^{15,16}$ showed response rates of $42 \%$ and $38 \%$, respectively, which suggested that the response rate was better than with epo alone. Recently, Negrin et al $^{17}$ published additional data from the American study, showing that around $50 \%$ of the patients with a response to the combination therapy lost their response when G-CSF was withdrawn, and some of these patients also regained a response when G-CSF was reintroduced. This strongly supports the hypothesis of a synergistic effect in vivo of G-CSF and epo.

The present study was designed as a randomized phase II trial to allow an unbiased selection of patients to one of two treatment alternatives. It had the following aims: to try to verify the response rate from the first Scandinavian study ${ }^{16}$ in an independant cohort of patients with MDS, to study whether a need for priming with G-CSF before epo was necessary, to study whether in vivo synergy between G-CSF and epo on 
erythroid response could be proven, and finally to study duration of response and long-term outcome. The answers to the first three questions were given by the results from the present study, while the fourth was approached using data from both the first and the second Scandinavian study.

\section{MATERIALS AND METHODS}

Patients. Patients in the randomized study were included from October 1992 to January 1995. All participating centers used uniform diagnostic criteria ${ }^{18}$ and the diagnosis was confirmed with two bone marrow samples over a period of at least 2 months. Disease duration was calculated from the date of the confirmatory bone marrow sample. Central pathologic review of bone marrow samples before the start of treatment and at the end of the study was performed by Chief Pathologist, Dr Å. Öst and copathologist, Dr L. Kanter-Lewensohn. All patients signed consent forms and the studies followed guidelines of the investigation review boards of Sweden and Norway.

Inclusion criteria were diagnosis of RA, RARS, or RAEB with either hemoglobin levels $<100 \mathrm{~g} / \mathrm{L}$ in combination with symptoms of anemia or transfusion-dependent anemia. Patients with active ongoing bleeding or transfusion-dependent thrombocytopenia were excluded from the trial. The long-term follow-up included in addition all evaluable patients from the first Scandinavian study, which has been previously described. ${ }^{16}$

Treatment. Patients were randomized to one of two alternatives; arm A started with G-CSF (filgrastim; Roche Pharmaceutical, Stockholm, Sweden) for 4 weeks and continued with the combination of G-CSF + epo (erythropoietin beta; Boehringer Mannheim, Stockholm, Sweden) for 12 weeks; arm B started with epo for 8 weeks and continued with the combination for 10 weeks. G-CSF and epo were self-administered subcutaneously (SC) and given daily. Treatment was started at the lowest dose and dose escalation was performed every 2 weeks, if necessary. In contrast to the previous Scandinavian study, doses were fixed and not given per kilogram body weight. Three dose levels of G-CSF ( 30 to 75 to $150 \mu \mathrm{g} / \mathrm{d}, \mathrm{SC}$ ) and two dose levels of epo $(5,000$ to $10000 \mathrm{U} / \mathrm{d})$ were used. A patient was considered evaluable for a response to G-CSF and epo if the combination was given for 6 weeks or more.

Sampling. Baseline hematologic parameters, lactate dehydrogenase, serum ferritin, serum epo, and soluble transferrin receptor were analyzed before and after treatment. Serum epo and transferrin receptor levels (serum tfr) were analyzed according to methods described by Wide et $\mathrm{al}^{19}$ and Beguin et al. ${ }^{20}$ Bone marrow sampling was performed before and after treatment and a cytogenetic analysis was made before treatment in all patients and after treatment in nine of these patients. The international prognostic scoring system (IPSS ${ }^{2}$ ) was used and a score was estimated for each patient.

Response criteria. A complete erythroid response was defined as an increase in hemoglobin to at least $115 \mathrm{~g} / \mathrm{L}$. A partial response (PR) was defined as an increase in hemoglobin with $15 \mathrm{~g} / \mathrm{L}$ or more in patients with nontransfused anemia and a $100 \%$ reduction of transfusion need in combination with stable hemoglobin level for $\geq 4$ weeks in those with pretreatment transfusion need. The aim of the G-CSF treatment was to obtain a neutrophil count of 6 to $10 \times 10^{9} / \mathrm{L}$ and values within this range were considered a complete response (CR). In patients who did not reach this range, the neutrophil counts were considered as PRs ( 3 to $6 \times$ $\left.10^{9} / \mathrm{L}\right)$, minor responses $\left(1\right.$ to $\left.3 \times 10^{9} / \mathrm{L}\right)$, or no response $\left(<1 \times 10^{9} / \mathrm{L}\right)$.

Maintenance treatment and long-term follow-up. Twenty-one evaluable patients were included in the first Scandinavian study on G-CSF + epo in MDS between November 1990 and $1992 .{ }^{16}$ Inclusion criteria were identical with those in the present study. A follow-up with regard to duration of response, survival, and progression to acute leukemia from start of treatment was made on these 21 patients and the 50 evaluable patients from the present study by the first of January 1997.
Patients in the follow-up analysis were thus included from November 1990 to January 1995, and the median follow-up from start of treatment was 43 months. All patients with an erythroid response in the second study were offered maintenance treatment with G-CSF and epo, while maintenance treatment in the first study was given on an individual basis.

Statistical analysis. Student's $t$-test, Mann-Whitney U-test, and analysis of variance (ANOVA) were used for comparison of continuous variables, whenever appropriate. $\chi^{2}$ analysis was used to compare categories. Kaplan-Meyer plots were used to describe survival, evolution to acute myeloid leukemia (AML), and duration of response.

\section{RESULTS}

Patient description. Fifty-six patients with a diagnosis of MDS were included in the study; 43 from Sweden and 13 from Norway. There were 30 men and 26 women. Median age was 69 years with a range from 48 to 87 years. Twenty-eight patients were randomized to each arm. Four patients were withdrawals; two developed acute myeloid leukemia during the interval between randomization and start of treatment, one patient was diagnosed with AML within 1 week from start of treatment (arm B), and one patient ( $\mathrm{arm} \mathrm{B}$ ) was diagnosed as having pyoderma gangrenosum after 4 weeks of combined treatment. Two patients were dropouts; one experienced a deterioration of a previously known depression within 2 weeks and refused further treatment and another one, living far from the hospital, refused to come to visits. Thus, 50 patients (26 men) were evaluable for a response to treatment and clinical characteristics of these patients are shown in Table 1. The median age in this group was 69.5 years (range, 48 to 87). Ten of these patients had RA, 13 RARS, and 27 RAEB. Fifteen patients had stable anemia and 35 were transfusion-dependent. The degree of transfusion need (units per month) was estimated over a period of 6 months in those with a disease duration over 6 months. In the rest, the minimum time for observation of transfusion need was 3 months. Each patient was transfused at the same hemoglobin level during the course of the study, but there was an interindividual variation between patients. Iron stain was positive in all patients. Serum creatinine was normal in all but three patients; one responder had a serum creatinine of $8 \%$ above the upper normal limit and two nonresponders had increases of $10 \%$ and $32 \%$, respectively. A cytogenetic analysis was done in 46 of the patients, 24 had a normal karyotype and 22 showed aberrations. Seven patients had deletion 5q-, with six as single abnormality. Ten patients had poor prognosis chromosomal patterns according to the risk score described by Greenberg et al. ${ }^{2}$ There were 46 patients evaluable for a IPSS score. Eleven had a low score, 19 an intermediate-1 score, 14 an intermediate- 2 score, and two a high score.

Three patients in arm B received only the epo and was thus not evaluable for a response to the combination treatment. These three patients were all nonresponders. Two of these, both with known heart disease, died of heart failure after 8 weeks of treatment and one patient showed disease progression and was withdrawn from treatment after 10 weeks. Thus, 47 patients were evaluable for a response to treatment with the combination of G-CSF and epo.

The absolute majority of the patients managed to selfadminister, while the rest needed support either with dosing or with dosing plus the injections. 
Table 1. Baseline Characteristics in 50 Evaluable Patients

\begin{tabular}{|c|c|c|c|c|}
\hline Variable & $\operatorname{Arm~A~n}=24$ & $\operatorname{Arm} B n=26$ & All Patients $n=50$ & $P$ Value \\
\hline Age (yr) & $69 \pm 10$ & $69 \pm 9$ & $69 \pm 9$ & .92 \\
\hline $\operatorname{Sex}(M / F)$ & $8 / 16$ & $18 / 8$ & $26 / 24$ & .01 \\
\hline RA & 4 & 6 & 10 & \\
\hline RARS & 8 & 5 & 13 & $.5^{*}$ \\
\hline RAEB & 12 & 15 & 27 & \\
\hline IPSS (low/lnt 1/Int 2/high)† & $4 / 10 / 7 / 2$ & $7 / 9 / 7 / 0$ & $11 / 19 / 14 / 2$ & .4 \\
\hline Disease duration (mos) & $11.6 \pm 11.7$ & $8.1 \pm 8.1$ & $9.3 \pm 9.8$ & .23 \\
\hline Prior RBC transfusions (yes/no) & $17 / 7$ & $18 / 8$ & $35 / 15$ & .90 \\
\hline RBC transf. units/moł & $2.5 \pm 1.1$ & $2.9 \pm 1.6$ & $2.7 \pm 1.4$ & .45 \\
\hline Hemoglobin level (g/dL) & $87.0 \pm 10.8$ & $86.3 \pm 12.8$ & $86.4 \pm 11.7$ & .85 \\
\hline ANC count $\times 10^{9} / \mathrm{L}$ & $2.2 \pm 1.9$ & $2.1 \pm 1.7$ & $2.2 \pm 1.8$ & .88 \\
\hline Platelet count $\times 10^{9} / \mathrm{L}$ & $210 \pm 141$ & $216 \pm 193$ & $213 \pm 168$ & .75 \\
\hline Bone marrow cellularity (\%) & $70 \pm 24$ & $78 \pm 20$ & $74 \pm 22$ & .23 \\
\hline Bone marrow blasts (\%) & $7.0 \pm 5.9$ & $6.2 \pm 3.9$ & $6.6 \pm 4.9$ & .58 \\
\hline Karyotype (normal/abnormal) & $13 / 10$ & $9 / 14$ & $22 / 24$ & .5 \\
\hline Serum erythropoietin (U/L)§ & $216(6-4979)$ & $237(20-4128)$ & 216 & .61 \\
\hline S. transferrin receptor $(\mathrm{ng} / \mathrm{mL})$ & $6,983 \pm 5,631$ & $6,480 \pm 5,987$ & $6,714 \pm 5,763$ & .77 \\
\hline
\end{tabular}

*Step-wise ANOVA.

†IPSS, International prognostic scoring system. ${ }^{2}$ Low, low risk; int-1, intermediate 1 risk; int-2, intermediate 2 risk; high, high risk. $\ddagger$ Transfusion needed from 35 patients with pretreatment RBC transfusions.

$\S M a n n-W h i t n e y U$ test.

Clinical results of treatment. Eighteen of the 47 fully evaluable patients (38\%) showed an erythroid response to treatment. Ten patients had a CR and eight a PR (Table 2). All nonresponders were exposed to the highest epo dose. Responses were not correlated to dose/body weight $(P>.5)$. Figure 1 shows hemoglobin levels during treatment in patients with a $\mathrm{CR}$. The response rates in the different subgroups of MDS were $20 \%, 46 \%$, and $37 \%$ for RA, RARS, and RAEB, respectively. The overall difference in response rate between subgroups was not statistically significant (Table 2). Only one response was observed in seven patients with 5q-. Forty-eight percent of the patients showed a complete neutrophil response to treatment (increase in absolute neutrophil count $[\mathrm{ANC}]$ to $\geq 6 \times 10^{9} / \mathrm{L}$ ) and $6 \%$ and $15 \%$, respectively, showed partial and minor reponses. Thirty-one percent of the patients were by definition nonresponders to G-CSF; $9 \%$ showed a decrease in neutrophil counts after treatment and $22 \%$ an increase less than $1 \times 10^{9} / \mathrm{L}$. Responses were not correlated to dose/body weight $(P>.5)$. All evaluable patients who were nonresponders to G-CSF received the highest dose. There were two patients with a minor response to G-CSF (ANC after treatment $<3 \times 10^{9} / \mathrm{L}$ ) who received only the low and intermediate G-CSF doses, respectively. Both of these patients showed increased thrombocytope-

Table 2. Erythroid Response to Treatment in 50 Evaluable Patients

\begin{tabular}{lcrrrrl}
\hline MDS Subtype & No. & NR & PR & CR & All R & $\%$ R \\
\hline RA & 10 & 8 & 1 & 1 & 2 & $20^{*}$ \\
RARS & 13 & 7 & 1 & 5 & 6 & $46^{*}$ \\
RAEB & 27 & 17 & 6 & 4 & 10 & $37^{*}$ \\
All patients & 50 & 32 & 8 & 10 & 18 & 38 \\
\hline
\end{tabular}

Note that three patients (all nonresponders) only were evaluable to treatment with epo alone.

Abbreviations: NR, no response; PR, partial response; $\mathrm{CR}$, complete response.

*No statistically significant differences in response rate $\left(\chi^{2}\right.$ analysis, $P=.43)$. nia during treatment, as well as an increase of bone marrow blasts at the final evaluation. No erythroid responses were seen in the group with decreased neutrophil counts after treatment, but otherwise the change in ANC was not associated with an erythroid response to treatment. Thirteen of 18 patients with an erythroid response to treatment received $70,000 \mathrm{U}$ of epo per week and five 35,000 U/week. One patient had a PR on the lower dose, but improved to a CR when the epo dose was increased after the study period. There was no correlation between the G-CSF dose and response to treatment; seven, three, and eight patients received the low, middle, and high dose, respectively.

There was no significant differences between the two randomization arms, apart from a significantly higher proportion of male patients in arm B (Table 1). The response rate was identical in the two arms. Nine of 24 patients $(38 \%)$ responded in $\operatorname{arm} \mathrm{A}(6 \mathrm{CR}, 3 \mathrm{PR})$ and 9 of $23(39 \%)$ in arm B (4 CR, 5 PR). There was clear evidence of in vivo synergy between G-CSF and epo in a proportion of the patients. Only 3 of the 9 responders in arm $\mathrm{B}$ showed any response to epo alone ( $1 \mathrm{CR}, 2$

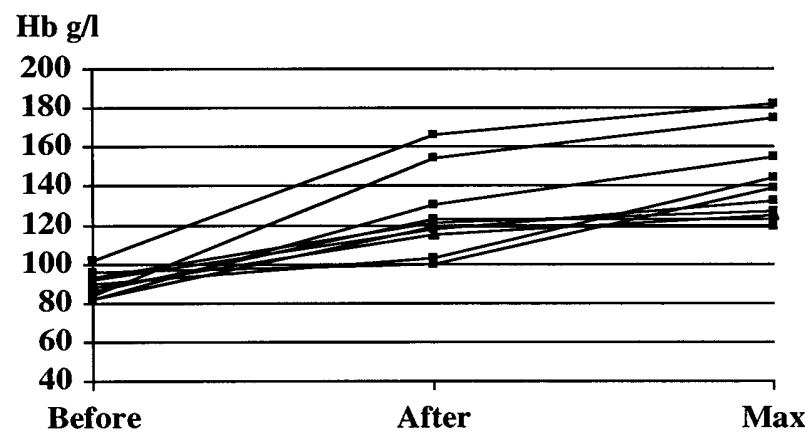

Fig 1. Hemoglobin levels in patients with a CR to treatment. Before, before start of treatment; after, at the end of the study period (16 weeks in arm A and 18 weeks in arm B); Max, maximum hemoglobin level during the maintenance phase. 
PR). An erythroid response induced by the addition of G-CSF was thus seen in 6 patients. Time from start of combination treatment to response did not differ between the two arms, but the study was not designed to analyze signs of early response.

Morphologic and laboratory parameters. All differences in percentages are given as percentage points. Treatment induced an overall increase in bone marrow cellularity $(+7 \%$ in nonresponders $(P=.06)$ and $+9 \%$ in responding patients $(P=$ $.06)$. The percentage of erythropoietic cells was generally reduced, $-9 \%$ in nonresponding and $-10 \%$ in responding patients $(P=.0009$ and .001 , respectively). The percentage of bone marrow blasts was unchanged in both groups of patients $(-1.3 \%$ in responders, $P=.25$ and $+0.4 \%$ in nonresponders, $P=.8$, Fig 1). Serum ferritin was significantly increased in nonresponders $(+582 \mu \mathrm{g} / \mathrm{L}, P=.0001)$, but slightly increased also in the responding patients $(+214 \mu \mathrm{g} / \mathrm{L}, P=0.10)$. Serum epo was increased after treatment in all patients (responders $+1,135 \mathrm{U} / \mathrm{L}, P=.22$ and nonresponders $+867 \mathrm{U} / \mathrm{L}, P=.05$ ). Mean corpuscular volume (MCV) was slightly increased in responding patients $(+4.2 \mathrm{fL}, P=.07)$, while no change was seen in nonresponding patients $(P=.53)$. Patients with RARS had higher serum soluble transferrin receptor levels (tfr) than the other subgroups $(P=.09$ with ANOVA for all three groups and $P=.05$ with $t$-test comparing RA and RARS). There was also a weak inverse correlation $(P=.049)$ between serum epo and serum tfr. Tfr levels were reduced in nonresponding patients $(-936 \mu \mathrm{g} / \mathrm{L}, P=.3)$, while it was increased in the responding population $(+2,582 \mu \mathrm{g} / \mathrm{L})$. However, because there was a large heterogeneity in the tfr response, this difference was not significant $(P=.34)$. Clonal evolution during treatment was observed in three patients in whom posttreatment karyotypic analyses were performed. Two patients with normal karyotype pretreatment showed $6 \mathrm{p}$ deletion and add $18 \mathrm{p}$ in a small proportion of the cells after treatment. One patient with $5 \mathrm{q}-$ before treatment showed, in addition, +8 in a few cells after treatment.

Adverse events. General side effects were few. Eight of the patients experienced minor flu-like side effects, which in the majority of cases, diminished after a couple of weeks. Irritation at local injection sites was observed in a few patients. Two patients developed increased splenomegaly. One of these had a long history of RARS with previous massive transfusion need and secondary hemochromatosis. The other had also RARS, and in addition to this, alcohol-induced liver cirrhosis and secondary splenomegaly. There was no general tendency of disease progression in the patient group, but three patients developed a significant increase in their bone marrow blasts (Fig 2).

The most important side effect was an overall decrease in platelet counts (Fig 3). This decrease was most pronounced in nonresponding patients $\left(-32 \times 10^{9} / \mathrm{L}, P=.004\right)$ and was a problem in patients with preexisting thrombocytopenia. Fourteen of 17 patients with pretreatment platelet counts of $<100 \times$ $10^{9} / \mathrm{L}$ showed a further decrease in their values after treatment. A reduction in platelet counts was also observed in the responding group (Fig 3), but this was mainly due to a decrease in patients with supranormal platelet levels before treatment.

Variables associated with a response to treatment. Table 3 shows continuous variables and Table 4 category variables in responding and nonresponding patients. Serum epo showed the strongest association with a response to treatment. A cut-off level of $500 \mathrm{U} / \mathrm{L}$ was more informative than $100 \mathrm{U} / \mathrm{L}$. The degree of RBC transfusion need was significantly associated with a response to treatment. The response rate in patients with $\geq 2 \mathrm{U}$ of RBC transfusions per month was $21.7 \%$ compared with $50 \%$ in those with less than $2 \mathrm{U} /$ month. Three variables showed borderline significance; pretreatment platelet counts, $\mathrm{MCV}$, and soluble transferrin receptor levels were higher in responding patients. The IPSS score showed no correlation with response rate. The response rates in the low, intermediate-1, and intermediate- 2 risk groups were $45 \%, 32 \%$, and $43 \%$, respectively. One of the two patients with a high risk score responded to treatment.

Long-term follow up and duration of response. Survival and time to progression to acute leukemia were calculated from start of treatment. The median time from final diagnosis to start of treatment was 6.5 months (range, 1 to 79 months). This variable had no association at all with response to treatment $(P>.5)$, response duration $(P>.5)$, or survival $(P>.5)$. However, the median time from diagnosis to treatment was shorter in patients developing AML during or after treatment (4.5 $v 8.5$ months, $P=.03$ ). The median survival time of the 71 evaluable patients in both studies was 26 months. Four patients were not scored according to IPSS due to missing cytogenetic data and thus 67 patients were given an IPSS score. Patients with a low risk score showed a survival of $68 \%$ at 5 years, while the median survival of patients with intermediate- 1 and intermediate- 2 risk score was 27 and 14 months, respectively. There were only two patients with a high score. Time to progression to AML was measured from start of treatment. Only one patient

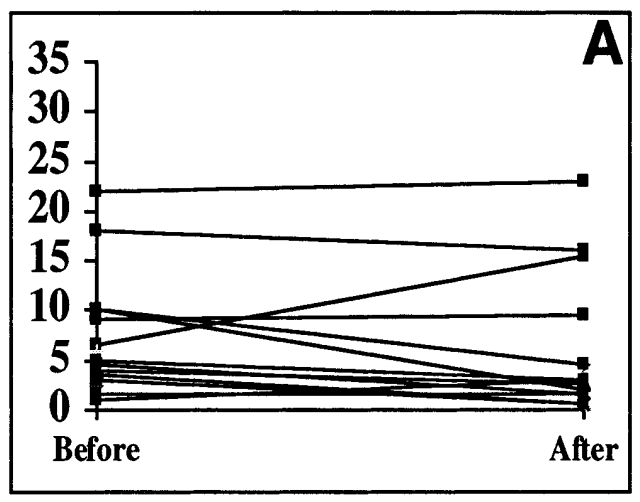

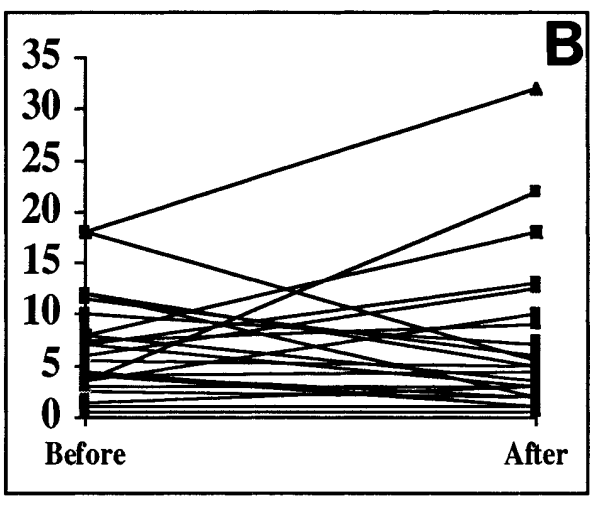

Fig 2. Bone marrow blasts (\%) before start of treatment and at the end of the treatment period in $(A)$ responding patients and (B) nonresponding patients. 
Fig 3. Platelet count $\left(\times 10^{9} / \mathrm{L}\right)$ before start of treatment and at the end of the treatment period in (A) responding patients and (B) nonresponding patients.
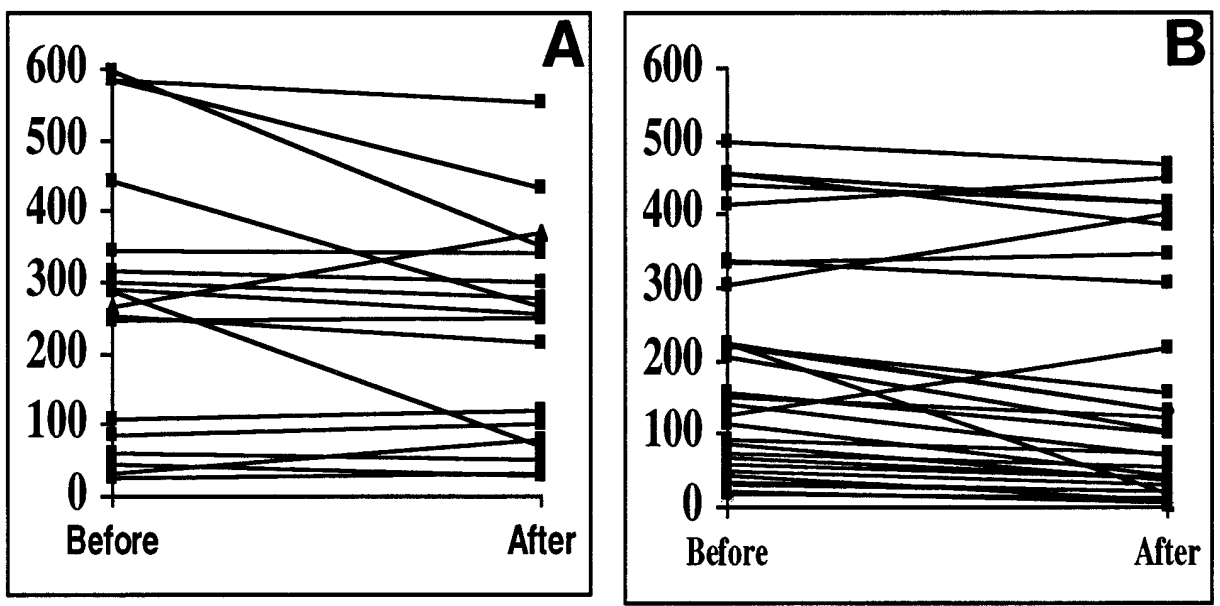

progressed to overt AML during the treatment period, but four additional patients developed AML within 2 months from the end of the study. Altogether 19 patients (28\%) progressed to AML during the observation period with a median time from start of treatment to progression of 11 months (range, 4 to 31 months). The frequency of leukemic progression was $12 \%$ ( 2 of 17 ) in the low-risk group, $21 \%$ (6 of 28 ) in the intermediate- 1 group, $45 \%$ (9 of 20 ) in the intermediate-2 group, and $100 \%$ (2 of 2) in the high-risk group.

Twenty patients of altogether 26 responders ( 8 in the previous and 18 in the present study) were given maintenance treatment. Three of the responding patients in the first study and three from the second study did not enter the maintenance phase. In the second study, one patient (PR) showed an increase of bone marrow blasts shortly after the first study period and was withdrawn from treatment, and two patients did not want to continue with the injections. The median time for duration of response was 24 months (range, 4 to 60 months) (Fig 4). Six of

Table 3. Continuous Variables in Responding and Nonresponding Patients

\begin{tabular}{lccc}
\hline \multicolumn{1}{c}{ Variable } & $\begin{array}{c}\text { Responding } \\
\text { Patients, } \\
\mathrm{n}=18\end{array}$ & $\begin{array}{c}\text { Nonresponding } \\
\text { Patients, } \\
\mathrm{n}=29\end{array}$ & PValue \\
\hline Age (yr) & $70 \pm 10$ & $68 \pm 10$ & .6 \\
Disease duration (mo) & $10 \pm 11$ & $9 \pm 10$ & .74 \\
RBC transf. units/mo* & $2.0 \pm 0.94$ & $3.0 \pm 1.4$ & .05 \\
Hemoglobin level (g/dL) & $88.6 \pm 7.1$ & $85.5 \pm 13.6$ & .37 \\
MCV (fL) & $90.4 \pm 17.5$ & $98.7 \pm 12$ & .09 \\
ANC count $\times 109 / \mathrm{L}$ & $1.9 \pm 1.5$ & $2.3 \pm 1.9$ & .46 \\
Platelet count $\times 109 / \mathrm{L}$ & $270 \pm 190$ & $182 \pm 148$ & .07 \\
Bone marrow cellularity (\%) & $68 \pm 21$ & $78 \pm 22$ & .13 \\
Bone marrow & & & .3 \\
$\quad$ erythropoiesis (\%) & $37 \pm 16$ & $31 \pm 20$ & .3 \\
Bone marrow blasts (\%) & $6.9 \pm 5.6$ & $6.4 \pm 4.6$ & .73 \\
Serum ferritin ( $\mu \mathrm{g} / \mathrm{L})$ & $515 \pm 284$ & $813 \pm 912$ & .18 \\
Serum lactate dehydro- & & & \\
$\quad$ genase & $7.0 \pm 1.8$ & $7.5 \pm 2.8$ & .56 \\
Serum erythropoietin $(\mathrm{U} / \mathrm{L})$ & $247 \pm 318$ & $1,293 \pm 1,531$ & .008 \\
S. transferrin receptor & & & \\
$\quad$ (ng/mL) & $8,667 \pm 6,972$ & $5,529 \pm 4,632$ & .07 \\
\hline
\end{tabular}

*Transfusion need in the 35 patients with pretreatment RBC transfusions. nine patients with RARS had a response duration of $\geq 18$ months. All patients showing a response duration of $\geq 12$ months had either a low or an intermediate-1 IPSS, but there was no difference between these two groups.

\section{DISCUSSION}

Treatment of anemia in MDS has so far been relatively discouraging. Epo alone shows an overall response rate of $20 \%$, with only around $10 \%$ responses in patients with preexisting transfusion need. Other treatment alternatives, such as low-dose cytosine arabinoside has shown an erythroid response rate of $30 \%$ or less, but with more side effects than the cytokines. ${ }^{21,22}$ The erythroid response rate in our study, 38\%, seems to be relatively high in comparison with other treatment alternatives for anemia in MDS and deserves further consideration.

The present study confirmed the response rate, $38 \%$, from the first Scandinavian study. These results are in accordance with another relatively large phase II study ${ }^{17}$ using the same drugs and also with a study using G-CSF and epo in combination with all-trans-retinoic acid (ATRA). ${ }^{23}$ The reason why two smaller studies have failed to show similar results is not clear, but might be due to the lower epo dose used in these two studies and maybe also the very high G-CSF dose used in the Japanese study. ${ }^{24,25}$

The second aim of the study was to investigate whether G-CSF treatment was needed as a primer before the addition of epo to obtain an optimal erythroid effect. This was appearantly not the case, as response rates in the two randomization arms were identical. The consequence of this result is that treatment with G-CSF and epo are started simultaneously in the ongoing third Scandinavian trial.

Third, the study aimed at showing evidence for in vivo synergy between G-CSF and epo. The response pattern in arm $\mathrm{B}$, starting with epo, showed that six of nine responders to combination treatment did not show any response to epo alone. Most responses to epo have been reported to occur within the first 8 weeks of treatment, ${ }^{11,26,27}$ and it is therefore not likely that all of these six patients would have developed a late response to epo as monotherapy. Moreover, some patients who maintained their transfusion need during the epo phase developed a pronounced increase in hemoglobin (hemoglobin $\geq 150 \mathrm{~g} / \mathrm{L}$ ) 
Table 4. Category Variables in Responding and Nonresponding Patients

\begin{tabular}{|c|c|c|c|c|c|}
\hline Variable & Favorable Group & Response Rate (\%) & Unfavorable Group & Response Rate (\%) & $P$ Value \\
\hline $\operatorname{Sex}(M / F)^{*}$ & Female & 38 & Male & 35 & .93 \\
\hline Karyotype (N/A)† & A & 41 & $\mathrm{~N}$ & 38 & .81 \\
\hline Pretreatment transfusions (yes/no) & no & 53.3 & yes & 28.6 & .09 \\
\hline Pretreatment transfusions U/month & $<2$ & 50 & $\geq 2$ & 21.7 & .04 \\
\hline Serum epo $<500 \mathrm{U} / \mathrm{L}$ or $\geq 500 \mathrm{U} / \mathrm{L}$ & $<500$ & 48.3 & $\geq 500$ & 15.8 & .02 \\
\hline Serum epo $<100 \mathrm{U} / \mathrm{L}$ or $\geq 100 \mathrm{U} / \mathrm{L}$ & $<100$ & 50 & $\geq 100$ & 29.4 & .18 \\
\hline
\end{tabular}

*Male/female.

†Normal karyotype/abnormal karyotype.

after the addition of G-CSF (Fig 1). Another argument for a true synergistic effect of the drugs was the high response rate, $46 \%$, in patients with RARS, as this MDS subgroup has shown a poor response to epo alone. ${ }^{12}$ Recently, additional evidence for a synergistic effect was published. Approximately $50 \%$ of the responders to G-CSF + epo in the American study ${ }^{17}$ lost their erythroid response when G-CSF was withdrawn. In our study, the synergistic effect seemed to be most pronounced in patients with RARS, even if patients with RAEB also seemed to respond relatively well to the combination $(37 \%)$. The response in patients with RA was not as good, (20\%), but the size of the RA group (10 patients) was too small to allow conclusions about the effect of the combination compared with epo alone. Only one of the seven patients with $5 \mathrm{q}$ - aberration showed a response to treatment. Four of these patients were found in the RA subgroup, which might explain the response rate in this group. The reason for the poor response rate in the $5 q$ - group is unclear. Serum epo levels and pretreatment transfusion need were comparable between patients with and without 5q-, which suggests that the poor response might be explained by the specific biology of this MDS subtype.

The fourth aim of the trial was to study clinical outcome and long-term efficacy. The median response duration of 24 months was comparable and even better than that described in the American study. ${ }^{17}$ Again, the most pronounced long-term efficacy was found in the RARS group with six of nine patients responding for more than 18 months. The observation that there are responses with a duration of more than 5 years is promising. Unfortunately, there are no data on long-term efficacy with epo alone, so that comparison can at present not be done. All patients, except one (data not shown), needed continuous maintenance treatment to maintain their response. It was also

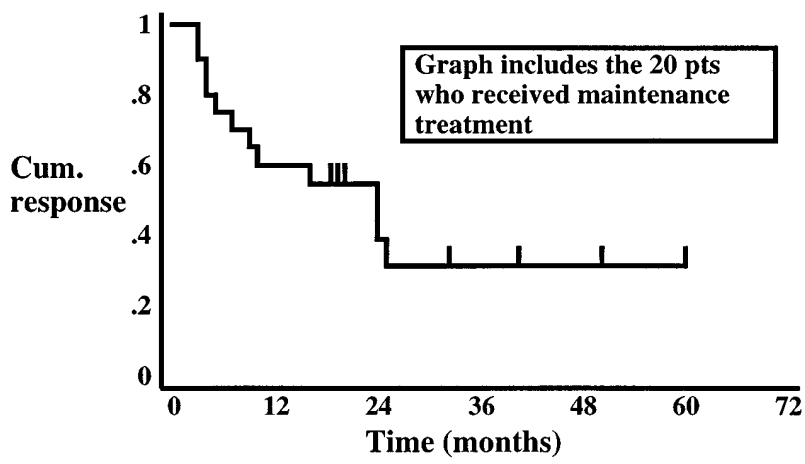

Fig 4. Duration of response in the 20 patients with primary response who entered maintenance phase. Median duration of response 24 months. found that a relatively high epo dose ( $\geq 50,000 \mathrm{U} / \mathrm{wk})$ was needed for the majority of the responding patients, but also that a minority of the responders managed to lower their epo dose to $30,000 \mathrm{U} / w \mathrm{w}$. However, dose reduction and titration of minimal effective doses were not a part of the protocol, but is included in the ongoing Scandinavian trial.

A recently published joint study by the Scandinavian and American Study Groups ${ }^{28}$ used multivariate analysis to study predictors of response in a larger patient sample (including 34 of the patients in the present study). Multivariate analysis was not repeated in the present study, but it was evident that the two predictors of response observed in the joint study, pretreatment serum epo levels and transfusion need, were significant univariate variables also in the present study. The most interesting additional finding was probably the higher median MCV value in responding patients and the fact that MCV actually increased during treatment in these patients. Macrocytosis has been defined as a dysplastic sign in $\mathrm{MDS}^{29}$ and the reason for an increase in responding patients is unclear. A previous study has shown that an erythroid response to G-CSF + epo is paralleled by both a reduced number of erythroid cells and a reduced number of apoptotic cells in the bone marrow, but how this is linked to the $\mathrm{MCV}$ findings remains to be investigated. ${ }^{30}$ Another finding was that pretreatment soluble serum transferrin levels were somewhat higher $(P=.07)$ in responding patients and also tended to increase more in responding than in nonresponding patients. This probably reflects a previously described finding that patients with RARS showed higher serum tfr values than other MDS subgroups. ${ }^{31}$

The IPSS score was not included as a variable in the previously published multivariate analysis, ${ }^{28}$ but from the present study, it was clear that it did not predict for a response to treatment. Similar results have been published for low-dose cytosine arabinoside (ara-C) $)^{21}$ and the present results support the hypothesis that variables predicting for survival are not always the same as those predicting for response to a specific treatment. The survival times in the present study seemed longer than those in the IPSS material. ${ }^{2}$ This could be explained by the fact that any clinical study implies a selection of patients and that our analysis included only evaluable patients. Whether treatment with G-CSF + epo could have a favorable impact on survival in certain patient groups remains to be investigated in a controlled trial.

Treatment with G-CSF and epo is well-tolerated and from the present study, there is no indication that it increases the number of bone marrow blasts or causes progression to acute leukemia. The lack of such a stimulating effect has previously been shown for G-CSF in a randomized study. ${ }^{5}$ However, treatment-induced 
thrombocytopenia has to be considered. Even if the reduction of platelet counts in most patients were moderate and without clinical risk, there were also cases showing significant reduction. This problem was basically only observed in nonresponding patients, which further supports ongoing attempts to develop a functioning predictive model.

The erythroid response rate of $38 \%$ for the anemia in MDS is promising in the aspect of previous treatment results. However, it is still a disappointment that almost two thirds of the patients are not responsive to this this type of treatment. A lack of response could partly be due to reasons that are associated with advanced disease, such as long-standing and high transfusion need or the finding of complex karyotype. However, specific biologic reasons cannot be excluded in some of the patients. Further studies on the biology of different subgroups of MDS might help to develop new treatment approaches.

In conclusion, the anemia associated with MDS of subtypes RA, RARS, and RAEB may respond to treatment with G-CSF and epo. Scandinavian and American data on hitherto 112 patients suggest that the response rate of approximately $40 \%$ is stable and not depending on a temporaray good selection of patients. There is a clear in vivo synergy between the two drugs, at least in patients with RARS, and long-term efficacy is so far promising. Considering the cost, it is necessary that ongoing studies focus on the indication for this treatment. A proposed model for selection of patients for this treatment has been recently published, ${ }^{28}$ but the model has to be prospectively validated before use outside clinical trials.

\section{REFERENCES}

1. Greenberg PL: The myelodysplastic syndromes, in Hoffman R, Benz E, Shattil S, Furie B, Cohen H (eds): Hematology: Basic Principles and Practice (ed 2). New York, NY, Churchill Livingstone, 1994, p 1098

2. Greenberg PL, Cox C, LeBeau M, Fenaux P, Morel P, Sanz G, Sanz M, Vallespi T, Hamblin T, Oscier D, Ohyashiki K, Toyama K, Aul C, Mufti G, Bennett J: International scoring system for evaluating prognosis in myelodysplastic syndromes. Blood 89:2079, 1997

3. Negrin R, Haeuber D, Nagler A, Kobayashi Y, Sklar J, Donlon T, Vincent M, Greenberg P: Maintenance treatment of patients with myelodysplastic syndromes using recombinant human granulocyte colony-stimulating factor. Blood 76:36, 1990

4. Yoshida Y, Hirashima K, Asano S, Takaku F: A phase II trial of recombinant human granulocyte colony-stimulating factor in the myelodysplastic syndromes. Br J Haematol 78:378, 1991

5. Greenberg P, Taylor K, Larson R, Koeffler P, Negrin R, Saba H, Ganser A, Jakubowski A, Gabrilove J, Mufti G, Cruz J, Hammond W, Broudy V, Langley GR, Keating A, Vardiman J, Lamborn K, Brown S: Phase III randomized multicenter trial of G-CSF vs observation for myelodysplastic syndromes (MDS). Blood 82:196a, 1993 (suppl 1)

6. Vadhan-Raj S, Keating M, LeMaistre A, Hittelman W, McCredie $\mathrm{K}$, Trujillo J, Broxmeyer H, Henney C and Gutterman J: Effects of recombinant human granulocyte-macrophage colony-stimulating factor in patients with myelodysplastic syndromes. N Engl J Med 317:1545, 1987

7. Shuster MW, Larson RA, Thompson JA, Coiffier B, Bennett JM, Israel RJ for the Shering-Plough/Sandoz MDS Study Group: Granulocyte-macrophage colony-stimulating factor (GM-CSF) for myelodysplastic syndrome (MDS): Results of a multi-center randomized controlled trial. Blood 76:318a, 1990
8. Shuster MW, Thompson JA, Larson RA, Dugan et al: Randomized phase II study of recombinant granulocyte-macrophage colonystimulating factor (RGM-CSF) in patients with neutropenia secondary to myelodysplastic syndrome (MDS). Blood 86:338a, 1995

9. Bessho M, Jinnai I, Matsuda A, Saito M, Hirashima K: Improvement of anaemia by recombinant eryhtropoietin in patients with myelodysplastic syndromes and aplastic anaemia. Int J Cell Cloning $8: 445,1990$

10. Hellström E, Birgegård G, Lockner D, Wide L, Helmers C, Öst Å: Treatment of myelodysplastic syndromes with recombinant human erythropoietin. Eur J Haematol 47:355, 1991

11. Rose EH, Abels RI, Nelson RA, McCullough DM, Lessin L: The use of r-HUEpo in the treatment of anaemia related to myelodysplasia (MDS). Br J Haematol 89:831, 1995

12. Hellström Lindberg E: Efficacy of erythropoietin in the myelodysplastic syndromes. An analysis of 205 patients in 17 studies. Br J Haematol 89:67, 1995

13. Amano Y, Koike K, Nakahata T: Stem cell factor enhances the growth of primitive erythroid progenitors to a greater extent than interleukin 3 in patients with aplastic anemia. Br J Haematol 85:663, 1993

14. Backx B, Broeders L, Löwenberg B: Kit ligand improves in vitro erythropoiesis in myelodysplastic syndromes. Blood 80:1213, 1992

15. Negrin RS, Stein R, Doherty K, Cornwell J, Vardiman J, Krantz S, Greenberg P: Treatment of the anaemia of myelodysplastic syndromes using human granulocyte-CSF in combination with erythropoietin. Blood 82:737, 1993

16. Hellström-Lindberg E, Birgegård G, Carlsson M, Carneskog J, Dahl IM, Dybedal I, Grimfors G, Merk K, Tangen JM, Winqvist I, Öst $\AA$ A: A combination of granulocyte-colony-stimulating factor and erythropoietin may synergistically improve the anaemia in patients with myelodysplastic syndromes. Leuk Lymphoma 11:221, 1993

17. Negrin RS, Stein R, Doherty K, Cornwell J, Vardiman J, Krantz S, Greenberg P: Maintenance treatment of the anemia of myelodysplastic syndromes with recombinant human granulocyte colony-stimulating factor and erythropoietin: Evidence for in vivo synergy. Blood 87:4076, 1996

18. Bennett JM, Catovsky D, Daniel MT, Flandrin G, Galton DAG, Gralnick HR, Sultan C, The French-American-British (FAB) Group: Proposals for the classification of the myelodysplastic syndromes. $\mathrm{Br} \mathbf{J}$ Haematol 51:189, 1982

19. Wide L, Bengtsson C, Birgegård G: Circadian rythm of human serum erythropoietin. Br J Haematol 51:189, 1989

20. Beguin Y, Clemons G, Pootrakul P, Fillet G: Quantitative assessment of erythropoiesis and functional classification of anemia based on measurements of serum transferrin receptor and erythropoietin. Blood 81:1067, 1993

21. Hellström-Lindberg E, Robèrt KH, Gahrton G, Forsblom AM, Lindberg G, Kock Y, Öst Å: A predictive model for the clinical response to low dose ara-C: A study of 102 patients with myelodysplastic syndromes or acute leukemia. Br J Haematol 81:503, 1992

22. Miller KB, Kyungman K, Morrison FS, Winter JN, Bennett JM, Neiman RS, Head DR, Cassileth PA, O'Connell MJ: The evaluation of low-dose cytarabine in the treatment of myelodysplastic syndromes: A phase-III intergroup study. Ann Hematol 65:162, 1992

23. Ganser A, Maurer A, Contzen C, Seipelt G, Ottmann OG, Schadeck-Gressel C, Kolbe K, Haas R, Zander C, Reutzel R, Hoelzer D: Improved multilineage response of hematopoiesis in patients with myelodysplastic syndromes to a combination therapy with all-transretinoic acid, granulocyte colony-stimulating factor, erythropoietin and alpha-tocopherol. Ann Hematol 72:237, 1996

24. Imamura M, Kobayashi M, Kobayashi S, Yoshida K, Mikuni C, Ishikawa Y, Matsumoto S, Sakamaki S, Niitsu Y, Hinoda Y, Yachi A, Kudoh T, Chiba S, Kasai M, Oka T, Okuno A, Maekawa I, Sakurada K, 
Miyazaki T: Failure of combination therapy with granulocyte colonystimulating factor and erythropoietin in myelodysplastic syndromes. Ann Hematol 68:163, 1994

25. Musto P, Falvone A, Carotenuto M: Granulocyte colonystimulating factor and erythropoietin for the anemia of myelodysplastic syndromes: A real improvement with respect to EPO alone? Blood 84:1687, 1994

26. Cazzola M, Mercuriali F, Brugnara C: Use of recombinant human erythropoietin outside the setting of uremia. Blood 89:4248, 1997

27. Stenke L, Wallvik J, Celsing F, Hast R: Prediction of response to treatment with human recombinant erythropoietin in myelodysplastic syndromes. Leukemia 7:1324, 1993

28. Hellström-Lindberg E, Negrin R, Stein R, Krantz S, Lindberg G,
Vardiman J, Öst Å, Greenberg P: Erythroid response to treatment with G-CSF plus erythropoietin for the anemia of patients with myelodysplastic syndromes: Proposal for a predictive model. Br J Haematol 99:344, 1997

29. May SJ, Smith SA, Jacobs A, Williams A, Bailey-Wood R: The myelodysplastic syndromes: Analysis of laboratory characteristics in relation to the FAB classifiacation. Br J Haematol 59:311, 1985

30. Hellström-Lindberg E, Kanter-Lewensohn L, Öst Å: Morphological changes and apoptosis in bone marrow from patients treated with granulocyte-CSF and erythropoietin. Leuk Res 21:415, 1997

31. Bowen DT, Culligan D, Beguin Y, Kendall R, Willis N: Estimation of effective and total erythropoiesis in myelodysplasia using serum transferrin receptor and erythropoietin concentrations, with automated reticulocyte parameters. Leukemia 8:151, 1994 